\title{
Identificar y responder preguntas resolubles mediante un diseño experimental: una experiencia con docentes de escuela primaria
}

\author{
Identifying and answering researchable questions through an \\ experimental design: an experience with primary school teachers
}

Valeria Carolina Edelsztein ${ }^{1}$

Lydia Raquel Galagovsky²

${ }^{1}$ Consejo Nacional de Investigaciones Científicas y Técnicas (CONICET), Buenos Aires, Argentina.
Autor correspondiente: valeriae@conicet.gov.ar
${ }^{2}$ Universidad de Buenos Aires (UBA), Facultad de Ciencias Exactas y Naturales, Buenos Aires, Argentina.

Resumen: La capacidad de identificar y responder preguntas investigables que puedan ser resueltas mediante un diseño experimental es considerada una competencia a ser desarrollada durante el aprendizaje en ciencias naturales. Lograr que los estudiantes cumplan con este objetivo necesariamente implica que el profesorado haya perfeccionado esta competencia previamente. Sin embargo, existen pocas investigaciones al respecto de cuán desarrollada se encuentra esta habilidad en docentes y prácticamente ninguna con la participación de maestros y maestras de escuela primaria. En este trabajo se presenta una secuencia de actividades didácticas en tres fases con el objetivo de indagar sobre esta habilidad, promover la capacidad de identificar, entre un genérico de preguntas investigables, aquellas que, efectivamente, podrían ser resueltas mediante un diseño experimental sencillo y luego formular un experimento adecuado, con variables mensurables y controladas, para responderlas. Dicha secuencia se implementó exitosamente con un grupo de 41 docentes de escuela primaria.

Palabras clave: Enseñanza de las ciencias; Escuela primaria; Preguntas investigables; Naturaleza de la ciencia; Diseño experimental educacional.

\begin{abstract}
The ability to identify and answer researchable questions that can be solved through experimental design is considered a competence to be developed while learning natural sciences; and getting students to attain this goal necessarily implies that teachers have previously perfected this competence. However, there is little research on how developed this ability is among teachers, and virtually none with the participation of primary school teachers. This paper presents a sequence of didactic activities in three phases with the aim of inquiring about this skill, of promoting the ability to identify, among a series of generic researchable questions, those that, indeed, could be solved through a simple experimental design and, then, of formulating a suitable experiment, with measurable and controlled variables, to answer them. This sequence was successfully implemented with a group of 41 elementary school teachers.

Keywords: Science education; Elementary school; Researchable questions; Nature of science; Experimental design.
\end{abstract}

Recebido em: 29/12/2020

Aprovado em: 26/03/2021 


\section{Introducción}

En las últimas décadas, han surgido distintas investigaciones que sostienen la importancia de promover en los estudiantes de ciencias naturales la competencia de formular preguntas investigables que puedan ser resolubles de manera empírica, mediante el dispositivo metodológico denominado diseño experimental (FERRÉS-GURT, 2017; FURMAN; BARRETO; PUIG, 2013; OECD, 2006; PATIÑO; VERA; MEISEL, 2010).

Sanmartí y Márquez (2012) han señalado que esta competencia no es una habilidad innata, ya que los sujetos no la desarrollarían de manera espontánea. Otros autores coinciden en que las dificultades para alcanzar la competencia de formular estrategias de diseño de experimentos van más allá de una mera comprensión conceptual acerca de los fenómenos sobre los que se desea indagar, ya que también están relacionadas con los procesos de construcción del conocimiento científico, tales como identificar cuáles son las variables de un experimento, establecer cómo controlarlas, cómo registrar datos y analizarlos, entre otras cuestiones (FERRÉS-GURT, 2017; FURMAN; BARRETO; PUIG, 2013; WINDSCHITL; THOMPSON; BRAATEN, 2008).

Es esencial, sin dudas, que esta competencia de formular diseños correctos para experimentos se desarrolle durante el aprendizaje específico de quienes los realizarán, pero podría resultar interesante, también, desarrollarla como una competencia dentro del ámbito de la ciencia escolar (ADÚRIZ-BRAVO; IZQUIERDO-AYMERICH, 2009). Sin embargo, se ha señalado que, en la enseñanza escolarizada en general, no suelen formularse preguntas que orienten hacia la construcción de dicha competencia, ya sea en el aula o a partir de los libros de texto (BLOSSER, 2000; CHIN; OSBORNE, 2008; COFRÉ et al., 2010; CONEJERA; JOGLAR; JARA, 2020; DAINES, 1986; DOMĖNECH CASAL, 2014; MAZZITELLI; GUIRADO; CHACOMA, 2011; MYHILL; DUNKIN, 2005; ROCA; MÁRQUEZ; SANMARTÍ, 2013).

Lograr que los estudiantes puedan identificar y formular este tipo de preguntas, necesariamente implica que el profesorado haya desarrollado esta competencia y que pueda explicitarla en su enseñanza pero, si bien existen investigaciones sobre cómo fomentarla en estudiantes (BULLOCK; ZIEGLER, 1999; DI MAURO; FURMAN; BRAVO, 2015; ZIMMERMAN, 2007; ZIMMERMAN; GLASER, 2001), hay pocos relevamientos realizados acerca del desarrollo de esta habilidad en docentes (CONEJERA; JOGLAR; JARA, 2020; FURMAN; PUIG; BARRETO, 2013) y ninguno de ellos en educación primaria.

En esta investigación se presenta el diseño de una serie de actividades de enseñanza con el objetivo de indagar las habilidades iniciales de maestros y maestras de escuela primaria para identificar preguntas investigables resolubles mediante un diseño experimental y promover el desarrollo de esta competencia. Su implementación se llevó a cabo con resultados satisfactorios en un grupo de 41 docentes en ejercicio de dos escuelas públicas, una de gestión estatal y otra de gestión privada.

\section{Marco teórico}

\section{Una reflexión epistemológica sobre el problema}

Numerosos investigadores sostienen la línea de trabajo sobre el aprendizaje de lo que identifican como preguntas investigables. Furman (2016), expresa al respecto 
que entre las capacidades que constituyen ese saber hacer de la ciencia, algunas son la identificación de problemas, la formulación de preguntas investigables, el planteo de hipótesis y predicciones, el diseño de experimentos, la recolección de datos, su interpretación y la elaboración de conclusiones. En coincidencia, Ferrés-Gurt (2017, p. 411 ) señala que "[...] cuando se habla de una pregunta investigable se hace referencia a la relación entre diferentes factores o fenómenos que puede ser investigada, que se puede responder recogiendo datos y analizándolos. [...]".

A partir de estas expresiones, parecería indicarse que el sintagma pregunta investigable refiere únicamente a aquellas que pueden ser resueltas de manera empírica, diseñando y ejecutando un experimento, recogiendo datos y analizándolos. Esto se ve reforzado por la clasificación de preguntas que realizan diversos autores. Por ejemplo, Harlen (2004) propone cuatro categorías para una clasificación de preguntas: aquellas que expresan sorpresa o interés, las que piden información, cuestiones filosóficas o complejas y, finalmente, preguntas investigables. Por su parte, García González y Furman (2014) proponen una clasificación en tres grupos: preguntas investigables - que proponen un interrogante susceptible de ser explorado empíricamente -, preguntas que indagan por una causa y preguntas acerca de un dato.

Todas estas caracterizaciones no establecen claras consideraciones acerca de la línea de investigación epistemológica sobre Naturaleza de la Ciencia (NOS, por sus siglas en inglés) (ADÚRIZ-BRAVO, 2005, 2011; IZQUIERDO-AYMERICH, 1996, 2000; MATTHEWS, 1994), ni discuten si habría diferencias para la aplicación de tales categorías en el área de la ciencia propiamente dicha, o en el área de ciencia escolar (ADÚRIZ-BRAVO; IZQUIERDO-AYMERICH, 2009; IZQUIERDO-AYMERICH; ADÚRIZ-BRAVO, 2003).

El marco de NOS ayuda a generar reflexiones acerca de la ciencia como actividad humana profundamente dinámica (IZQUIERDO-AYMERICH, 1996, 2000) y a abordar la producción de conocimiento desde su complejidad e historicidad, en contraposición a las ideas de 'verdades científicas' como productos acabados (ADÚRIZ-BRAVO, 2011). Desde esta perspectiva, llamar preguntas investigables solo a aquellas que se resuelven mediante un diseño experimental, estaría sosteniendo una visión particular sobre el quehacer científico, bajo la idea de que únicamente los experimentos donde se controlan variables mensurables serían los aceptados en ciencia. Esa mirada reduccionista de la ciencia es cuestionada desde la perspectiva epistemológica actual, que sostiene que toda ciencia es investigable mediante metodologías específicas y particulares que permiten modelizar y argumentar, basadas en evidencias fácticas que requieren articularse con creatividad e imaginación (ADÚRIZ-BRAVO, 2005; IZQUIERDO-AYMERICH; ADÚRIZ-BRAVO, 2003). Por dar ejemplos, tanto en lingüística como en paleontología o en economía, se investiga y se concluye sin hacer experimentos con control de variables; por lo tanto, hay 'preguntas investigables' en diferentes ciencias que no se responderían mediante un diseño experimental.

Por tales motivos, en esta investigación se distinguirá entre la generalidad de preguntas investigables y aquellas preguntas resolubles mediante un diseño experimental (PRmE) y se definirá que la competencia de producir una PRmE incluye el diseño apropiado de un experimento con variables mensurables y controladas para responderla. 


\section{Las características de una PRmE}

Más allá de clasificaciones y de las consideraciones presentadas en el apartado previo, varios autores han profundizado en la definición de una pregunta investigable. Por ejemplo, Sanmartí y Márquez (2012) indican que formular una pregunta investigable requiere identificar qué es una variable, poder realizar un diseño experimental de modo que permita distinguir entre aquellas que varían y las que deben controlarse y definir un protocolo de recolección de datos. En esta misma línea, Cerda (2007) agrega que deben ser factibles de ser contestadas dentro de un lapso de tiempo relativamente corto, ser atrapantes, sencillas, y llevar a la reflexión y a la posibilidad de generar nuevas preguntas. Dibarboure y Rodríguez (2013) indican adicionalmente que las preguntas investigables no pueden responderse con lo que ya se conoce acerca de un tema, deben incluir un conflicto capaz de ser abordado, capacidad de hipotetizar al respecto, variables y la búsqueda de su respuesta requerirá de lo que denominan "procesos del quehacer científico": observar, experimentar, procesar información, analizar, inferir y deducir.

Domènech Casal (2014), por otra parte, se centra en cómo identificar a las preguntas investigables. Comenta que, en general, las preguntas que empiezan con "¿Por qué...?" o "¿Cómo...?" no serían investigables, dado que no es posible diseñar una metodología de obtención de datos para responderlas. Roca, Márquez y Sanmartí (2013) coinciden en esta apreciación. Asimismo, indican que las preguntas que empiezan con "¿Qué sucede si...?" o "¿Se observa alguna diferencia si...?" son, efectivamente, investigables porque permiten plantear hipótesis y planificar diseños experimentales. En cambio, Kelsey y Steel (2001) sostienen que las preguntas investigables plantean una comparación específica que puede ser puesta a prueba, por ejemplo: "¿Vuelan los pájaros más rápidamente en días de sol o cuando llueve?"

Para el diseño de la secuencia didáctica implementada en esta investigación, y sobre la base de las publicaciones dentro de la línea de investigación de preguntas investigables, se consideró que las características para identificar una PRmE son las siguientes:

a. Debe ser lo suficientemente específica como para poder establecer algunas variables de medición en un experimento (DOMÈNECH CASAL, 2014; KELSEY; STEEL, 2001; ROCA; MÁRQUEZ; SANMARTÍ, 2013; SANMARTÍ; MÁRQUEZ, 2012.

b. Su redacción debe ser clara en cuanto a la calidad de las variables a medir (SANMARTÍ; MÁRQUEZ, 2012).

c. Su redacción no debe referirse a la búsqueda de definiciones de términos ni debe poder responderse recordando información sino mediante procesos que impliquen identificar problemas, tomar decisiones, analizar datos y evaluarlos (FERRÉS-GURT, 2017; GARCÍA GONZÁLEZ; FURMAN, 2014; HARLEN, 2004).

\section{El Modelo de Aprendizaje Cognitivo Consciente Sustentable (MACCS)}

Las actividades de enseñanza de la secuencia didáctica presentada en este trabajo se encuentran enmarcadas en las recomendaciones didácticas del Modelo de Aprendizaje Cognitivo Consciente Sustentable (MACCS) (GALAGOVSKY, 2004a, 2004b). Este Modelo propone que cada sujeto demandará recibir nueva información si ha tomado conciencia sobre su necesidad de completar, cerciorar o apuntalar aquellos 
conceptos que está construyendo; la aparición de esta motivación, surgirá, sobre todo, al reconocer diferencias entre sus representaciones mentales y las de otros participantes. De esta manera, un aprendizaje sustentable es reflexivo, y permite al sujeto, en argumentación con otros, seleccionar de entre todos sus potenciales conceptos inclusores (NOVAK, 1999), aquellos conceptos sostén correctos.

Este modelo propone que un conflicto cognitivo surge en un sujeto cuando al menos dos piezas de información claramente contradictorias entre sí se procesan simultáneamente en su memoria de trabajo (ERICSSON; SIMON, 1999; JOHNSTONE, 1997), sembrando dudas porque el sujeto las percibe como potencialmente correctas. Para lograr esto, el desarrollo de la clase debe destinar un tiempo, no solamente a mostrar las ideas previas de los sujetos - como se propone en el paradigma del cambio conceptual (POSNER et al., 1982) -, sino también a relevar sus orígenes y argumentaciones soporte, en un ambiente distendido donde se valore la diversidad de representaciones mentales que los sujetos exhiben para justificar sus elecciones.

Se requiere, por lo tanto, un diseño didáctico apropiado que efectivice la toma de conciencia sobre dicha diversidad de información respecto de representaciones mentales y argumentos. El resultado es la motivación de cada sujeto para buscar información fidedigna que refuerce su argumentación o que satisfaga su incertidumbre (GALAGOVSKY, 2004a, 2004b). En este caso, se propone generar en los participantes la necesidad de lograr una definición consensuada para definir PRmE, como parte de la Fase I de la secuencia didáctica.

\section{Las preguntas en la enseñanza}

La evidencia disponible indica que, en la enseñanza escolarizada en general, los docentes no suelen promover instancias de experimentación en las aulas ni formular PRmE. En general, se centran en procesos memorísticos, con pocos experimentos - que buscan demostrar un principio y concepto -, y no en la problematización de los contenidos; la gran mayoría de las preguntas formuladas por docentes están orientadas a respuestas de descripción y generalización (BARNES; BRITTON; TORBE, 1986; BULLAUDE et al., 2008; BUSQUETS; SILVA; LARROSA, 2016; CHIN; OSBORNE, 2008; COFRÉ et al., 2008; CONEJERA; JOGLAR; JARA, 2020; DOMÈNECH CASAL, 2014; GUIRADO; MAZZITELLI; OLIVERA, 2013; MAZZITELLI; GUIRADO; CHACOMA, 2011; PASMANIK; CERÓN, 2005; ROCA; MÁRQUEZ; SANMARTÍ, 2013).

Sin embargo, si comparamos cómo fue modificándose esta tendencia a lo largo del tiempo, encontramos que, mientras que en los estudios piloto de Daines (1986) se registró que el $93 \%$ de las preguntas de docentes de nivel primario y secundario apelaban únicamente a la memorización de contenidos, quince años más tarde, Blosser (2000) notó que, pese a que la mayoría de las preguntas de los profesores de ciencias seguían buscando identificar si el estudiante sabía o no una dada información y estaban centradas en la memorización de conceptos, el porcentaje había disminuido hasta un $60 \%$. Resultados similares obtuvieron Myhill y Dunkin (2005) al analizar las preguntas de docentes de nivel primario. Esto podría darnos un indicio de cierto interés por parte del profesorado en modificar sus hábitos de enseñanza en relación a las preguntas formuladas en el contexto áulico y es, a partir de este interés, que surge la propuesta de una secuencia didáctica específica para mejorar la competencia docente en la identificación de PRmE. 


\section{Objetivos}

El objetivo general de este trabajo fue analizar si, a partir de una secuencia didáctica específica, es posible mejorar en docentes de escuela primaria la competencia de identificar PRmE de entre un grupo de preguntas investigables y de realizar, con ellas, el diseño - y eventual ejecución - de una investigación escolar, mediante experimentos con control de variables.

Los objetivos específicos de esta investigación fueron:

- Relevar las principales ideas previas de los docentes acerca de las características que debe presentar una pregunta para ser considerada PRmE.

- Consensuar una definición de PRmE entre los participantes a partir del intercambio y del desarrollo de contenido teórico por parte de las docentes del taller.

- Fomentar en los y las maestras la competencia de construcción de un diseño experimental. explicitando el control de variables, las formas de medición y el análisis de los datos.

- Evaluar si se produce un progreso en la capacidad de los participantes de identificar PRmE a raíz de la propuesta didáctica.

- Conocer las opiniones de cada participante al finalizar el taller para analizar el impacto potencial en sus hábitos de enseñanza.

\section{Metodología}

Las actividades se implementaron en cuatro talleres de capacitación con un total de 41 docentes en ejercicio de dos escuelas primarias públicas de la Ciudad de Buenos Aires, una de gestión estatal y una de gestión privada. Cada taller constó de 3 encuentros de 2 horas cada uno. Los docentes se dividieron en cuatro grupos según la disponibilidad de las instituciones:

- Grupo A: 8 docentes en el contexto de escuela de gestión estatal.

- Grupo B: 6 docentes en el contexto de escuela de gestión estatal.

- Grupo C: 15 docentes en el contexto de escuela de gestión privada.

- Grupo D: 12 docentes en el contexto de escuela de gestión privada.

La investigación realizada incluyó tres fases con cada grupo.

La Fase I tuvo como objetivo crear la necesidad de definir qué es una PRmE. En primera instancia se solicitó a los docentes - sin darles información previa alguna - la elección de preguntas PRmE de entre las 10 de la tabla 1. Los grupos (A-D) mostraron los porcentajes de elecciones correctas que se indican en la tabla $\mathbf{2}$. A continuación, se fomentó la aparición de conflictos cognitivos - en el marco del MACCS -, solicitando que expusieran sus criterios personales de clasificación de dichas preguntas, mediados por una discusión de tal forma de lograr la construcción, en conjunto, de una definición consensuada de PRmE (cuadro 1).

La Fase II tuvo como objetivo desarrollar en los docentes la competencia de generar un diseño experimental. Para ello, las PRmE identificadas fueron disparadoras para que los docentes realizaran un diseño de experimentación explicitando el control de variables, formas de medición, de consideración de errores y de posterior análisis de los datos. Esta actividad podía resolverse tanto desde la perspectiva de una ciencia escolar, como desde una perspectiva sofisticada enmarcada en un contexto científico real. 
La Fase III permitió evaluar la efectividad de la propuesta didáctica.

A continuación, se describen detalladamente las tres fases de la propuesta, los instrumentos didácticos utilizados y sus resultados.

\section{Propuesta didáctica: fases y resultados}

\section{Fase I: caracterización de preguntas hasta consenso sobre PRmE}

Al comenzar la primera jornada, se les repartió a los docentes una hoja impresa con 10 preguntas (ver columna izquierda de la tabla 1) y, sin proporcionar información alguna, se les solicitó que señalaran aquellas que, desde sus ideas y opiniones, pudieran ser 'resolubles mediante experimentos' (PRmE). Los resultados de tales elecciones, sobre los 41 docentes totales, se muestran en la columna derecha de la tabla 1.

Las preguntas 2, 4, 8, 9 y 10, marcadas en negrita en la tabla 1 son PRmE por cumplir con los criterios mencionados en el apartado de Marco Teórico. Las razones para no considerar PRmE a las otras preguntas se indican a continuación.

La pregunta 1 (¿Por qué late el corazón?) es inconmensurable y, por lo tanto, no cumple el criterio (a), pues su resolución implica una modelización y no un experimento.

La pregunta 3 (¿Es mejor lavarse las manos con jabón que solo con agua?) no cumple el criterio (b): para resolverla sería imprescindible acordar qué quiere decir el término mejor. ¿Se refiere a un menor número de microorganismos en las manos al finalizar el lavado?, ¿a una menor tasa de enfermedad de los sujetos que se lavan las manos? Sin esta especificación no es posible diseñar un dispositivo experimental que permita darle respuesta.

Las preguntas 5 (¿Qué reacción química se observa en un clavo oxidado?) y 7 (¿Qué es la fotosíntesis?) buscan una definición, por lo tanto, no cumplen con el criterio (c).

La pregunta 6 (¿Pueden las semillas germinar en cualquier tipo de suelo?) sería perfectamente investigable pero su generalidad la vuelve irresoluble con un experimento; no cumple el criterio (b). Para ser PRmE debería, por ejemplo, especificarse el tipo de semillas a utilizar y los tipos de suelo a ensayar.

Tabla 1 - Preguntas a ser clasificadas por los docentes $(n=41)$ como resolubles mediante experimentos (PRmE) durante la fase I

\begin{tabular}{|l|c|}
\hline \multicolumn{1}{|c|}{ Preguntas de la Indagación (Fase I) } & $\begin{array}{c}\text { \% de } \mathbf{4 1} \text { docentes que señalaran } \\
\text { a la pregunta como PRmE }\end{array}$ \\
\hline 1. ¿Por qué late el corazón? & 73 \\
\hline 2. ¿Influye la presencia de agua en la velocidad de oxidación de un clavo? & 93 \\
\hline 3. ¿Es mejor lavarse las manos con jabón que solo con agua? & 93 \\
\hline 4. ¿En qué posición hay que ubicarse respecto del sol para ver un arcoíris cuando llueve? & 88 \\
\hline 5. ¿Qué reacción química se observa en un clavo oxidado? & 93 \\
\hline 6. ¿Pueden las semillas germinar en cualquier tipo de suelo? & 85 \\
\hline 7. ¿Qué es la fotosíntesis? & 78 \\
\hline 8. ¿Qué relación hay entre la cantidad de luz que recibe una planta y su crecimiento? & 83 \\
\hline 9. ¿Cómo cambia la frecuencia cardíaca después de correr cinco minutos? & 100 \\
\hline 10. ¿Cuánta sal hay que agregarle a un litro de agua para percibir el gusto salado? & 76 \\
\hline
\end{tabular}

Las respuestas consideradas apropiadas se indican en negrita. En la columna derecha se muestra qué porcentaje de docentes indicó cada pregunta como PRmE.

Fuente: elaboración de las autoras. 
Los porcentajes de la tabla 1 corresponden a las elecciones sobre el total de los docentes $(n=41)$. Los resultados provenientes de cada uno de los grupos fueron similares (datos no mostrados) y, por lo tanto, se consideró reunir los datos de todos los grupos.

Para verificar que esto podía realizarse, se calculó también el porcentaje promedio de preguntas correctamente seleccionadas como PRmE para el total de docentes y para cada grupo durante esta fase. Para el total de docentes, este valor fue del 51\% (si hubieran seleccionado correctamente todas las PRmE como tales, tendrían un $100 \%$ ). Para cada grupo, los valores se muestran en la tabla $\mathbf{2}$. Se ve que, en todos los casos, también son valores cercanos al $50 \%$.

Por lo tanto, a partir de este momento, se analizarán los datos de todos los grupos en conjunto.

Tabla 2 - Resultados de las indagaciones de las fases I

\begin{tabular}{|c|c|c|}
\hline Grupo & $\begin{array}{c}\text { Cantidad de } \\
\text { docentes }\end{array}$ & $\begin{array}{c}\text { \% Promedio de PRmE } \\
\text { correctamente identificadas }\end{array}$ \\
\hline A & 8 & 49 \\
\hline B & 6 & 53 \\
\hline C & 15 & 52 \\
\hline D & 12 & 51 \\
\hline
\end{tabular}

Se indican los porcentajes promedios de preguntas correctamente seleccionadas como PRmE para los grupos A a D.

Fuente: elaboración de las autoras.

Una vez completada por escrito la elección individual de cuáles preguntas de la tabla 1 fueron consideradas PRmE o no, se procedió a leer en voz alta cada pregunta y se estimuló a los docentes a que compartieran sus ideas y justificaran sus criterios de clasificación. Esta actividad - que fue grabada para su posterior análisis-intentaba promover la aparición de conflictos cognitivos individuales, en atención a la toma de conciencia sobre la variedad de respuestas y de argumentos para sostenerlas. Lograr estos conflictos cognitivos desencadenaría la motivación por recibir más información acerca de la validez de las elecciones y/o ideas propias de cada sujeto.

Tal como se esperaba, y se observa en la tabla 1, todas las opciones tuvieron un alto porcentaje de elección. Durante la puesta en común los docentes señalaron que sus elecciones respondían a criterios tales como: que las preguntas fueran interesantes, que no se respondieran desde el conocimiento común, que fueran relevantes para la ciencia, que puedan responderse con mediciones, que den resultados concretos, que no sean peligrosas. Estos argumentos, que se repitieron en todos los grupos, se anotaron en el pizarrón a medida que fueron surgiendo y se muestran en la columna izquierda del cuadro 1.

A continuación, se presentaron algunos aspectos teóricos relacionados con la NOS y diversas clasificaciones teóricas sobre preguntas científicas (GARCÍA GONZÁLEZ; FURMAN, 2014; HARLEN, 2004; KELSEY: STEEL, 2001). Al retomar los argumentos iniciales, se resolvieron conflictos cognitivos individuales, llegando a consenso para construir la columna derecha del cuadro 1. 
Cuadro 1 - Criterios de los docentes para identificar e definir PRmE

\begin{tabular}{|c|c|}
\hline $\begin{array}{c}\text { Criterios iniciales de los docentes para } \\
\text { identificar PRmE }\end{array}$ & Criterios consensuados para definir una PRmE \\
\hline $\begin{array}{l}\text { - Que sean interesantes. } \\
\text { - Que no sean de conocimiento común. } \\
\text { - Que sean relevantes para la ciencia. } \\
\text { - Que puedan hacerse mediciones. } \\
\text { - Que den resultados concretos. } \\
\text { - Que no sean peligrosas. }\end{array}$ & $\begin{array}{l}\text { - Que plantee un problema susceptible de ser } \\
\text { resuelto mediante un experimento (es decir, que } \\
\text { no refiera a búsqueda de términos o definiciones). } \\
\text { _ } \quad \text { Que su redacción incluya variables relacionadas } \\
\text { con el problema a resolver. } \\
\text { _ Que sea específica. }\end{array}$ \\
\hline
\end{tabular}

La columna izquierda muestra los criterios iniciales que plantearon los docentes para la identificación de una PRmE, y la columna derecha los criterios finales consensuados para su definición.

Fuente: elaboración de las autoras.

Al finalizar la Fase I, el consenso acerca de cuáles de las preguntas de la tabla 1 eran PRmE fue unívoco y el cuadro 1, con la comparación de los criterios iniciales y los consensuados, quedó plasmado en el pizarrón.

Esto dio pie al desarrollo de la Fase II, orientado propiamente a la construcción de un diseño experimental y los factores que debieran tenerse en cuenta al llevarlo a la práctica, ya fuera en un contexto científico o en el aula.

\section{Fase II: la competencia de construcción de un diseño experimental}

Durante la segunda jornada del taller, cada grupo se dividió en pequeños subgrupos de 3 a 4 participantes y se les dio a elegir alguna de las PRmE de la tabla 1 para desarrollar un diseño experimental que permitiera llegar a responderla. Las preguntas elegidas por los diversos grupos fueron las 8, 9 y 10 de la tabla 1. Durante la tercera jornada, cada grupo expuso su diseño y se discutieron las variables elegidas en cada caso.

Algunos de los aspectos más relevantes que se suscitaron durante las discusiones fueron los siguientes.

- Pregunta 8: ¿Qué relación hay entre la cantidad de luz que recibe una planta y su crecimiento?

Cuatro subgrupos eligieron esta pregunta para realizar el diseño experimental.

En general todos los equipos plantearon un diseño muy similar: trabajarían con dos plantas, una a la que mantendrían en un lugar oscuro (por ejemplo, adentro de un armario) y otra que quedaría expuesta a la luz natural. Es destacable que todos los grupos indicaron que trabajarían con plantas de la misma especie e, idealmente, del mismo tamaño, y que controlarían que el nivel de riego fuera el mismo para ambas. Sin embargo, solamente dos de los grupos especificaron cómo medirían el crecimiento: uno de ellos dijo que registraría la altura de la planta con una regla y otro de los grupos señaló que contaría el número de hojas. Tres grupos indicaron que harían un registro fotográfico 'para comparar'. Ninguno de los grupos especificó cada cuánto haría el registro.

Durante la discusión, además del análisis de todas las variables mencionadas por los subgrupos, se debatió la ambigüedad remanente del concepto de crecimiento, ya que también podría considerarse el peso total de la planta o el crecimiento de ramas 
o de raíces laterales o centrales, en cuyos casos deberían utilizarse más plantas como población a ser investigada, con la consecuente necesidad de realizar estadísticas en las mediciones respectivas. Así mismo, surgió la pregunta de si no deberían agregarse plantas expuestas a luz artificial, por la ambigüedad remanente del concepto luz. Las discusiones pusieron en evidencia las dificultades inherentes a la precisión de la variable crecimiento y de su medición, y su relación con la especificidad de las conclusiones que podrían deducirse del experimento diseñado.

- Pregunta 9: ¿Cómo cambia la frecuencia cardíaca después de correr cinco minutos? Cinco subgrupos eligieron esta pregunta para realizar el diseño experimental.

También en este caso el diseño fue muy similar para todos los equipos. Coincidieron en que deberían medir la frecuencia cardíaca de una misma persona antes y después de correr cinco minutos y que sería importante repetir el experimento con varias personas (con sugerencias de 5 a 15 personas, según el subgrupo). Tres subgrupos indicaron que contarían el número de latidos por minuto. Un grupo señaló que contaría el número de latidos por 30 segundos y luego multiplicaría el valor por 2. Un equipo no indicó cómo realizaría la medición. Dos equipos señalaron que sacarían un valor promedio de la frecuencia cardíaca de todos los participantes antes y después de correr para comparar los valores.

Durante la discusión grupal surgió la pregunta acerca de si era conveniente que los participantes fueran todos varones o mujeres, si debían tener una edad similar o si era una variable que no era necesario controlar. También se discutió si convendría realmente obtener un promedio de la frecuencia cardíaca de los participantes o si era mejor comparar las diferencias individuales. Estas discusiones pusieron también en evidencia las dificultades inherentes a la precisión de la variable frecuencia cardíaca en relación a cualidades del sujeto estudiado, y su vínculo con la especificidad de las conclusiones que podrían deducirse.

- Pregunta 10: ¿Cuánta sal hay que agregarle a un litro de agua para percibir el gusto salado?

Dos subgrupos eligieron esta pregunta para realizar el diseño experimental. Ambos plantearon que prepararían diferentes recipientes con la misma cantidad de agua y cantidades crecientes de sal y se las darían a probar a distintas personas para que indicaran cuándo percibían el gusto salado. Uno de los grupos especificó qué utilizaría 1, 2, 4 y 6 gramos de sal por litro de agua pero que podría repetir el experimento si fuera necesario ajustar los valores. Ninguno de los grupos especificó el número de participantes con el que trabajarían ni la necesidad de establecer grupos control o placebos para comparar.

De forma similar a lo ocurrido en la pregunta de la frecuencia cardíaca, durante la discusión grupal surgió el interrogante de si convendría elegir a los participantes en función de su edad, de si eran o no fumadores, de su género, etc. También se planteó cuál sería el valor inicial y la magnitud de los intervalos crecientes de concentración. Las discusiones se enriquecieron con la necesidad de considerar grupos de sujetos con características específicas para llegar a conclusiones válidas a partir de una variable de medición dicotómica (respuesta si/no), pero subjetiva, como es el percibir (o no) gusto salado. 
Luego de las presentaciones de cada grupo (y a raíz de ellas) se discutió con los docentes cómo plantear nuevas PRmE a partir de diferentes situaciones que podrían darse en el contexto del aula.

Para reforzar conceptos y lograr la competencia de plantear y resolver una PRmE, durante la tercera jornada del taller se propusieron dos acciones cotidianas: (1) regar las plantas y (2) notar que la reja del balcón se está oxidando. Se les pidió a los docentes que reconstruyeran las PRmE respectivas y que plantearan un diseño experimental apropiado que involucrara cada acción.

Los docentes plantearon adecuadamente PRmE para el primer caso (por ejemplo, ¿Afecta al crecimiento de la planta regarla todos los días respecto de hacerlo día por medio?), pero tuvieron dificultades en el segundo caso. Ejemplos de PRmE para esta situación serían: ¿Cambia la velocidad de oxidación de la reja si se deja a la intemperie respecto de si se cubre? o ¿Depende la velocidad de oxidación de la reja del nivel de humedad en el ambiente? En ambos casos es posible plantear un diseño experimental para resolver el interrogante. En cambio, los docentes postularon el interrogante: ¿Cómo se produce químicamente una oxidación? Esta no es una PRmE ya que no es posible diseñar una metodología de obtención de datos para responderla, sino que requiere gran cantidad de estudios y modelizaciones. Es posible inferir que, dado que el fenómeno de oxidación es más complejo, la capacidad de formular PRmE se ve disminuida por la necesidad primera de los docentes de comprender el fenómeno en cuestión, tal como han señalado Furman, Barreto y Puig (2013) y Ferrés-Gurt (2017).

\section{Fase III: indagación final}

Antes de finalizar la tercera jornada, se les repartió a los docentes una hoja impresa que constaba de 10 nuevas preguntas con el objetivo de que nuevamente marcaran aquellas que pueden considerarse como PRmE. Dichas preguntas se muestran en la columna izquierda de la tabla 3 . En la columna derecha se indica qué porcentaje del total de docentes señaló cada pregunta como PRmE.

Tabla 3 - Preguntas a ser clasificadas por los docentes como resolubles mediante experimentos (PRmE), durante la fase III

\begin{tabular}{|l|c|}
\hline \multicolumn{1}{|c|}{ Preguntas de la Indagación (Fase III) } & $\begin{array}{c}\text { \% de } \\
\text { a la docentes que señalaran } \\
\text { pregunta como PRmE }\end{array}$ \\
\hline 1. ¿Cuánto tiempo dura el sangrado si nos pinchamos la punta del dedo con un alfiler? & 95 \\
\hline 2. ¿Cómo funciona un detergente? & 0 \\
\hline 3. ¿Por qué cuando tocamos metal lo sentimos más frío que la madera? & 12 \\
\hline 4. ¿Qué ocurre con la comida cuando llega al estómago? & 17 \\
\hline 5. ¿Cambia la velocidad de un autito si se desliza sobre arena o sobre baldosas? & 85 \\
\hline 6. ¿Es posible disolver diez sobres de azúcar en 100 mL de agua hirviendo? & 100 \\
\hline 7. ¿Por qué un coco flota, pero una piedra se hunde en agua? & 0 \\
\hline 8. ¿Conviene usar film o jugo de limón para evitar que la fruta se oscurezca al cortarla? & \\
\hline 9. ¿Pueden $\mathbf{2}$ gramos de vitamina C por día prevenir los resfriados? & 93 \\
\hline 10. ¿Por qué el agua del mar es salada y la del río no? & 88 \\
\hline
\end{tabular}

Las respuestas consideradas apropiadas se indican en negrita. En la columna derecha se muestra qué porcentaje del total de docentes indicó cada pregunta como PRmE.

Fuente: elaboración de las autoras. 
Las preguntas 1, 5, 6, 8 y 9, marcadas en negrita en la tabla 3 son PRmE por cumplir con los criterios consensuados. Tal como se discutió con las preguntas destacadas durante la Fase II, algunas de ellas requerirían establecer ciertas variables al momento de diseñar un experimento. Por ejemplo, la pregunta 1 (¿Cuánto tiempo dura el sangrado si nos pinchamos la punta del dedo con un alfiler?) precisaría establecer las características de los individuos sobre los que se realizaría la medición (edad, condiciones de salud, calidad del pinchazo, etc.). Las restantes preguntas no se consideran PRmE dado que ninguna cumple el criterio (a), pues su resolución implica una modelización y no un experimento.

Al analizar las respuestas individuales se observó que, luego del desarrollo de la propuesta didáctica, las preguntas que ofrecieron mayor dificultad para una clasificación correcta como PRmE fueron: ¿Cambia la velocidad de un autito si se desliza sobre arena o baldosas? (pregunta 5) y ¿Pueden 2 gramos de vitamina C por día prevenir los resfriados? (pregunta 9) mientras que las más señaladas como PRmE sin serlo fueron: ¿Por qué cuando tocamos metal lo sentimos más frío que la madera? (pregunta 3) y ¿Qué ocurre con la comida cuando llega al estómago? (pregunta 4).

\section{Capacidad de identificar PRmE antes y después de la propuesta didáctica}

Antes de la propuesta didáctica, el porcentaje promedio de preguntas correctamente seleccionadas como PRmE por los docentes fue de 51\%. Luego de la propuesta didáctica, este porcentaje promedio se elevó al 93\%. Es decir que la capacidad de identificar PRmE mejoró de manera significativa.

Además de comparar simplemente los porcentajes promedio de respuestas correctas, es posible realizar un análisis más fino de los resultados utilizando otros parámetros para comparar la calidad de las mismas. En los problemas de clasificación (en este caso, discernir entre PRmE y aquellas que no lo son), los resultados (las respuestas de los docentes), se suelen separar en dos clases: positivos y negativos. En este caso, el 'positivo' es la capacidad de identificar las PRmE de entre una variedad de preguntas. Esto se debe a que, en este contexto, se consideraría más grave que frente a una pregunta no PRmE los docentes pensaran que sí lo es (y que, por lo tanto, infieran que podrían responderla mediante un diseño experimental). Existen, entonces, cuatro tipos de respuestas posibles:

- Verdaderos positivos: casos positivos que el docente identifica como tales (es decir, PRmE que el docente identifica correctamente como PRmE).

- Verdaderos negativos: casos negativos que el docente identifica como tales (es decir, no PRmE que el docente identifica correctamente como no PRmE).

- Falsos negativos (también conocidos como errores de tipo I): casos positivos que el docente identifica como negativos, (PRmE que el docente identifica como no PRmE).

- Falsos positivos (también conocidos como errores de tipo II): casos negativos que el docente identifica como positivos, (No PRmE que el docente identifica como PRmE).

Para evaluar la calidad de las respuestas, se puede utilizar el parámetro de precisión, que intenta responder qué proporción de identificaciones positivas fue correcta, es decir, cuántas preguntas de las que identificaron como PRmE realmente lo 
eran. Los valores de precisión oscilan entre 0 y 1 . Así, un docente que no responde con falsos positivos tiene una precisión de 1.

Para los 41 docentes antes y después de la intervención didáctica, los valores de precisión medidos como la fracción de 'positivos' detectados fueron de 0,51 (Indagación Fase I) y 0,94 (Indagación Fase III). La amplia diferencia entre estos valores da indicios claros de la efectividad de la propuesta didáctica para el desarrollo de habilidades de identificación de PRmE entre un conjunto más genérico de preguntas.

\section{Reflexión metacognitiva de los docentes}

En esta última etapa del taller, también se les pidió a los docentes participantes que expresaran su opinión escrita acerca de las actividades realizadas. Las opiniones se agruparon en cinco categorías y los resultados se muestran a continuación como porcentajes (tabla 4). El total supera el $100 \%$ porque algunas de las 41 opiniones fueron clasificadas en más de una categoría.

Tabla 4 - Relevamiento de las opiniones escritas por cada participane del taller (asignación a cinco categorías)

\begin{tabular}{|l|c|}
\hline Categorías provenientes de las opiniones escritas de los participantes de los talleres \\
\hline $\begin{array}{l}\text { 1. Nunca antes había desarrollado un diseño experimental. } \\
\text { 2. Me di cuenta de que las preguntas que formulo en clase apuntan a recordar de memoria y no están orientadas a } \\
\text { indagar mediante diseños experimentales. }\end{array}$ & 63 \\
\hline $\begin{array}{l}\text { 3. Voy a intentar incorporar experimentos/experiencias a mis clases. } \\
\text { temas que tengo que dar. }\end{array}$ & 39 \\
\hline \begin{tabular}{l} 
5. Siempre pensé que necesitaba un laboratorio para poder hacer experimentos en clase y ahora veo que no. \\
\hline
\end{tabular} & 17 \\
\hline
\end{tabular}

Fuente: elaboración de las autoras.

Un $63 \%$ de los participantes manifestó que nunca antes había pasado por la experiencia de desarrollar un diseño experimental a partir de una pregunta (Categoría 1). Un $44 \%$ valoró la propuesta porque le sirvió para notar que las preguntas en sus clases eran más bien memorísticas y cerradas (Categoría 2); un 39\% sugirió que a partir de la secuencia implementada intentaría incorporar este tipo de actividades en sus aulas (Categoría 3) y un $22 \%$ consideró que promoverlas podría despertar la motivación de sus estudiantes (Categoría 4). Un 17\% sostuvo que esta propuesta le sirvió para entender que no es necesario que la escuela cuente con un laboratorio para poder desarrollar indagaciones experimentales con sus estudiantes (Categoría 5). No se registraron opiniones desfavorables.

A continuación, se muestran cinco transcripciones textuales - a modo de ejemplo - de respuestas escritas completas, representativas de las categorías mencionadas en la tabla 4:

- Es la primera vez que tengo que pensar en cómo hacer un experimento. Fue divertido y pensé que me iba a costar más. Ahora quiero hacerlo con mis alumnos. [Categorías 1 y 3].

- Nunca había pensado en las preguntas que hago en clase y me di cuenta de que son todas para responder de memoria sobre lo que yo enseño o lo que dice el manual. [Categoría 2].

- Me sirvió el taller porque tuve que sentarme a pensar que las preguntas que yo hago no son para investigar y cómo debería cambiar para que despierten el interés de mis alumnos y podamos hacer experimentos. Voy a tratar de usarlo para el próximo tema. [Categorías 2 y 3]. 
- Estuvo bueno. Quiero probar hacer algunas de estas preguntas con mis alumnos porque creo que va a hacer que se entusiasmen y no se aburran. [Categorías 3 y 4].

- Me gustó hacer este taller porque siempre pensé que necesitaba un laboratorio y mucho material específico para hacer experimentos, algo que no tengo en mi escuela. Pero ahora vi que se puede hacer de otra manera así que voy a tratar de probarlo con mis chicos. Seguramente los motive más. [Categorías 3, 4 y 5].

\section{Conclusiones}

Dado que distintos grupos de investigación en didáctica de las ciencias promueven la competencia de 'formular preguntas investigables' (CHIN; OSBORNE, 2008; FERRÉS-GURT, 2017; FURMAN; BARRETO; PUIG, 2013; PATIÑO; VERA; MEISEL, 201; ROCA; MÁRQUEZ; SANMARTÍ, 2013; WINDSCHITL; THOMPSON; BRAATEN, 2008) y teniendo en cuenta que en el marco de la ciencia escolar resulta difícil realizar experimentos con control de variables - pues el contexto escolar es de poca experimentación, los recursos de laboratorio frecuentemente escasos, o, existen clases de laboratorio con una lógica protocolizada, para que se arribe a un 'resultado correcto' -, se consideró valioso proponer una secuencia didáctica con docentes de escuela primaria en ejercicio para construir la competencia de identificar y responder 'preguntas resolubles mediante experimentos' (PRmE).

Sobre esta base, el objetivo del trabajo fue explorar las habilidades iniciales de un grupo de docentes para identificar PRmE, llegar a un consenso para distinguir este tipo de preguntas respecto de un genérico de preguntas que pueden hacerse desde la ciencia $y$, posteriormente, realizar un diseño experimental apropiado. La experiencia fue realizada con 41 docentes de escuela primaria divididos en cuatro talleres que se extendieron a lo largo de tres jornadas de 2 horas cada uno.

Los resultados medidos en función de las respuestas iniciales y finales a cuestionarios ad hoc, tanto en porcentajes como en valores de precisión, mostraron que la propuesta resultó exitosa para el desarrollo de la competencia de identificar PRmE. Esto es relevante debido a que prácticamente no existen investigaciones en las que se indague cuán desarrollada se encuentra esta habilidad en docentes de escuela primaria y se presenten actividades para su mejora.

Por otra parte, a partir de la reflexión metacognitiva de los docentes participantes, se observó que, para muchos de ellos, era la primera vez que se enfrentaban al cuestionamiento respecto del tipo de preguntas que plantean en sus clases y también a la necesidad de desarrollar un diseño experimental. Otros tantos, reconocieron que nunca habían evaluado la posibilidad de llevar adelante un diseño experimental por no contar con un laboratorio en sus establecimientos de enseñanza. Más de un tercio de los docentes involucrados expresaron su deseo de llevar este tipo de actividades a las aulas.

Es interesante destacar que la habilidad de formular una PRmE y/o el diseño apropiado de una experimentación con control de variables resultó más complejo que la mera identificación de una $\mathrm{PRmE}$, ya que la ausencia del conocimiento científico subyacente actúa como un factor limitante. Este dato resulta interesante pues sitúa a la competencia de aprender a formular PRmE en paralelo a contextos de aprender contenidos científicos. Esto significa que desde la ciencia escolar se abre la importante oportunidad de fomentar este tipo de competencias en los estudiantes, discutiendo acerca de los procedimientos de la ciencia erudita para la obtención de conocimientos 
específicos, y no simplemente, enseñando esos conocimientos específicos como si hubieran sido revelados cual verdades inapelables.

\section{Referencias}

ADÚRIZ-BRAVO, A. Desde la enseñanza de los "productos de la ciencia" hacia la enseñanza de los "procesos de la ciencia" en la universidad. Río Cuarto: Universidade Nacional de Río Cuarto, 2011. Recuperado el 21 jul. 2021 de: https://cutt.ly/3m9oNiL.

ADÚRIZ-BRAVO, A. Una introducción a la naturaleza de la ciencia: la epistemología en la enseñanza de las ciencias naturales. Buenos Aires: Fondo de Cultura Económica, 2005.

ADÚRIZ-BRAVO, A.; IZQUIERDO-AYMERICH, M. Un modelo de modelo científico para la enseñanza de las ciencias naturales. Revista Electrónica de Investigación en Educación en Ciencias, Buenos Aires, v. 4, n. 1, p. 40- 49, 2009. Recuperado el 21 jul. 2021 de: https://cutt.ly/HQyfJWv.

BARNES, D. R.; BRITTON, J. N.; TORBE, M. Language, the learner and the school. Chicago: Penguin, 1986.

BLOSSER, P. E. How to ask the right questions. Arlington: NSTA Press, 2000.

BULLAUDE, M. G.; CORDOBA, L. M.; TORRES, M. C.; DE MORAN, J. A. Análisis de metodologías de estudio en química inorgánica. Formación Universitaria, La Serena, Chile, v. 1, n. 6, p. 29-34, 2008. DOI: https://doi.org/fmsvrg.

BULLOCK, M.; ZIEGLER, A. Scientific reasoning: developmental and individual differences. In: F.E. WEINERT, F. E.; SCHNEIDER, W. (ed.). Individual development from 3 to 12: findings from the Munich longitudinal study. Cambridge: Cambridge University Press, 1999. p. 38-54.

BUSQUETS, T.; SILVA, M.; LARROSA, P. Reflexiones sobre el aprendizaje de las ciencias naturales: nuevas aproximaciones y desafíos. Estudios Pedagógicos, Valdivia, Chile, v. 42, p. 117-135, 2016. (Número especial). DOI: https://doi.org/gpsh.

CERDA, H. La investigación formativa en el aula, la pedagogía como investigación. Bogotá: Magisterio, 2007.

CHIN, C.; OSBORNE, J. Students' questions: a potential resource for teaching and learning science. Studies in Science Education, London, v. 44, n. 1, p. 1-39, 2008. DOI: https://doi.org/btn5np.

COFRÉ, H.; CAMACHO, J.; GALAZ, A.; JIMÉNEZ, J.; SANTIBÁÑEZ, D.; VERGARA, C. La Educación científica en Chile: debilidades de la enseñanza y futuros desafíos de la educación de profesores de ciencia. Estudios Pedagógicos, Valdivia, Chile, v. 36, n. 2, p. 279-293, 2010. DOI: https://doi.org/ gpsj.

CONEJERA, A.; JOGLAR, C.; JARA, R. Promoviendo la formulación de buenas preguntas en la clase de biología en secundaria: una propuesta didáctica a partir de situaciones problema. Ciência \& Educação, Bauru, v. 26, p. 1-17, 2020. DOI: https://doi.org/10.1590/1516-731320200034.

DAINES, D. Are teachers asking higher level questions? Education, USA, v. 106, n. 4, p. 368-374, 1986.

DI MAURO, M. F.; FURMAN, M.; BRAVO, B. Las habilidades científicas en la escuela primaria: un estudio del nivel de desempeño en niños de 4to año. Revista Electrónica de Investigación en Educación en Ciencias, Buenos Aires, v. 10, n. 2, p. 1-11, 2015.

DIBARBOURE, M.; RODRÍGUEZ, D. (ed.). Pensando en la enseñanza de las ciencias naturales: la pregunta investigable. Montevideo: Camus, 2013.

DOMĖNECH CASAL, J. Indagación en el aula mediante actividades manipulativas y mediadas por ordenador. Alambique, Barcelona, v. 76, p. 17-27, 2014. 
ERICSSON, K. A.; SIMON, H. A. Protocol analysis: verbal reports as data. 3. ed. Massachusetts: MIT Press, 1999.

FERRÉS-GURT, C. El reto de plantear preguntas científicas investigables. Revista Eureka sobre Enseñanza y Divulgación de las Ciencias, Cádiz, v. 14, n, 2, p. 410-426, 2017. Recuperado el 21 jul. 2021 de: https://cutt.ly/nm27a99.

FURMAN, M. Educar mentes curiosas: la formación del pensamiento científico y tecnológico en la infancia. Buenos Aires: Fundación Santillana, 2016. Recuperado el 21 jul 2021 de: https://cutt.ly/ rm28L3c.

FURMAN, M.; BARRETO, M.; PUIG, N. El procés d'aprendre a plantejar preguntes investigables. Educació Química, Barcelona, v. 14, p. 11-18, 2013. DOI: https://doi.org/10.2436/20.2003.02.102.

GALAGOVSKY, L. Del aprendizaje significativo al aprendizaje sustentable, parte 2: derivaciones comunicacionales y didácticas. Enseñanza de las Ciencias, Barcelona, v. 22, n. 3, p. 349-364, $2004 a$.

GALAGOVSKY, L. Del aprendizaje significativo al aprendizaje sustentable, parte 1: el modelo teórico. Enseñanza de las Ciencias, Barcelona, v. 22, n. 2, p. 230-240, 2004b.

GARCÍA GONZÁLEZ, S. M.; FURMAN, M. G. Categorización de preguntas formuladas antes y después de la enseñanza por indagación. Praxis y Saber, Bogotá, v. 5, n. 10, p. 75-91, 2014. DOI: https://doi.org/10.19053/22160159.3023.

GUIRADO, A.; MAZZITELLI, C.; OLIVERA, A. Representaciones sociales y práctica docente: una experiencia con profesores de física y de química. Revista de Orientación Educacional, Valparaíso, Chile, v. 27, n. 51, p. 87-105, 2013.

HARLEN, W. Teaching, learning and assessing. London: Paul Chapman, 2004.

IZQUIERDO-AYMERICH, M. Fundamentos epistemológicos. In: PERALES PALACIOS, F. J.; CAÑAL DE LEON, P. (coord.). Didáctica de las ciencias experimentales: teoría y práctica de la enseñanza de las ciencias. Alcoy: Marfil, 2000. p. 35-64.

IZQUIERDO-AYMERICH, M. Relación entre la historia y la filosofía de la ciencia y la enseñanza de las ciencias. Alambique, Barcelona, v. 8, p. 7-21, 1996.

IZQUIERDO-AYMERICH, M.; ADÚRIZ-BRAVO, A. Epistemological foundations of school science. Science \& Education, Dordrecht, v. 12, n. 1, p. 27-43, 2003. DOI: https://doi.org/b3q9x8.

JOHNSTONE, A. H. Chemistry teaching: science or alchemy? Journal of Chemical Education, Washington, v. 74, n. 3, p. 262-268, 1997. DOI: https://doi.org/10.1021/ed074p262.

KELSEY, K.; STEEL, A. The truth about science. Arlington: NSTA Press, 2001.

MATTHEWS, M. R. Science teaching: the role of history and philosophy of science. NewYork: Routledge, 1994.

MAZZITELLI, C.; GUIRADO, A.; CHACOMA, M. S. La docencia y la enseñanza de las ciencias: análisis de las representaciones de profesores. Revista de Orientación Educacional, Valparaíso, Chile, n. 48, p. 77-94, 2011.

MYHILL, D.; DUNKIN, F. Questioning learning. Language and Education, London, v. 19, n. 5, p. 415427, 2005. DOI: https://doi.org/10.1080/09500780508668694.

NOVAK, J. D. Conocimiento y aprendizaje: los mapas conceptuales como herramientas facilitadoras para escuelas y empresas. Madrid: Alianza, 1999.

OCDE. PISA 2006: marco de la evaluación: conocimientos y habilidades en ciencias, matemáticas y lectura [Madrid]: OCDE, 2006. Recuperado el 21 jul. 2021 de: https://cutt.ly/4QygRKK. 
PASMANIK, D.; CERÓN, R. Las prácticas pedagógicas en el aula como punto de partida para el análisis del proceso enseñanza-aprendizaje: un estudio de caso en la asignatura de química. Estudios Pedagógicos, Valdivia, Chile, v. 31, n. 2, p. 71-87, 2005. DOl: https://doi.org/d3k9v9.

PATIÑO, L.; VERA, Á.; MEISEL, J. Análisis de la práctica docente desde una experiencia de la enseñanza de las ciencias basada en la indagación. Educere, Caracas, v. 49, n. 14, p. 333-334, 2010. Recuperado 21 jul. 2021 de: https://cutt.ly/wQygvD2.

POSNER, G. J.; STRIKE, K. A.; HEWSON, P. W.; GERTZOG, W. A. Accommodation of a scientific conception: toward a theory of conceptual change. Science Education, Hoboken, v. 66, n. 2, p. 211-227, 1982. DOI: https://doi.org/10.1002/sce.3730660207.

ROCA, M.; MARQUEZ, C.; SANMARTÍ, N. Las preguntas de los alumnos: una propuesta de análisis. Enseñanza de las Ciencias, Barcelona, v. 31, n. 1, p. 95-114, 2013. DOI: https://doi.org/gpv4.

SANMARTÍ, N.; MÁRQUEZ, C. Enseñar a plantear preguntas investigables. Alambique, Barcelona, v. 70, p. 27-36, 2012.

WINDSCHITL, M.; THOMPSON, J.; BRAATEN, M. Beyond the scientific method: Model-based inquiry as a new paradigm of preference for school science investigations. Science Education, Hoboken, v. 92, n. 5, p. 941-967, 2008. DOI: https://doi.org/10.1002/sce.20259.

ZIMMERMAN, C. The development of scientific thinking skills in elementary and middle school. Developmental Review, New York, v. 27, n. 2, p. 172-223, 2007. DOI: https://doi.org/fc6hzq.

ZIMMERMAN, C.; GLASER, R. Testing positive versus negative claims: a preliminary investigation of the role of cover story in the assessment of experimental design skills. Los Angeles: California University, 2001. Recuperado el 27 jul. 2021 de: https://files.eric.ed.gov/fulltext/ED462412.pdf. 